Annals of Pure and Applied Mathematics

Vol. 13, No. 1, 2017, 99-117

ISSN: 2279-087X (P), 2279-0888(online)

Published on 28 February 2017

www.researchmathsci.org

DOI: http://dx.doi.org/10.22457/apam.v13n1a10

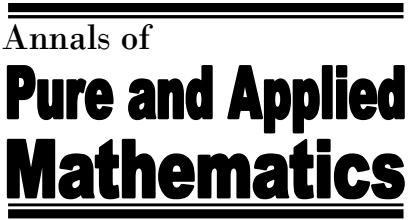

\title{
On Second Geometric-Arithmetic Index and Co-PI Index of Special Chemical Molecular Structures
}

\author{
Wei Gao, Li Liang and Yuhua Chen
}

School of Information Science and Technology, Yunnan Normal University, Kunming 650500, China. Email: gaowei@ynnu.edu.cn

Received 17 January 2017; accepted 15 February 2017

\begin{abstract}
In theoretical chemistry, chemical compounds and drugs are modelled as graphs, and the second geometric-arithmetic index and Co-PI index have been introduced to measure the stability of alkanes and the strain energy of cycloalkanes. These indices have found several applications in QSPR/QSAR study, and also used to test the toxicity of drugs. In this paper, we present the computational formulas for calculating the second geometric-arithmetic index and Co-PI index of unilateral polyomino chain, unilateral hexagonal chain, V-phenylenic nanotubes and nanotori, and hexagonal triangle graph. In addition, the general second geometric-arithmetic index and general Co-PI index are defined, and the representations of special chemical molecular graphs in general version are derived.
\end{abstract}

Keywords: molecular graph, second geometric-arithmetic index, Co-PI index, unilateral polyomino chain, unilateral hexagonal chain, V-phenylenic nanotubes and nanotori, hexagonal triangle graph

\section{AMS Mathematics Subject Classification (2010): $05 \mathrm{C} 15$}

\section{Introduction}

In chemical engineering, one of the most important applications of theoretical chemistry is measuring chemical, physical, medicine and pharmaceutical properties of molecules which is called alkanes. Large number of indices depending on the graphical structure of the alkanes were defined and employed to model both the melting point and boiling point of the molecules. There are several vertex distance-based and degree-based indices which are introduced to analyze the chemical properties of molecule graph and applied in a variety of QSPR/QSAR studies. For instance: Wiener index, PI index, Szeged index, geometricarithmetic index and atom-bond connectivity index are introduced to test the performance of chemical molecular structures. There are several papers contributing to determining the indices of special molecular graphs (See Yan et al., [1], Gao and Shi [2], Xi and Gao [3], Dou et. al., [4], and Gao and Wang [5] for more details).

All (molecular) graphs considered in this article are finite, loopless, and without multiple edges. Let $G$ be a (molecular) graph with vertex set $V(G)$ and edge set $E(G)$ . The notations and terminologies used but undefined in this paper can refer to [6].

By considering the degrees of vertices in $G$, Vukicevic and Furtula [7] introduced 
Wei Gao, Li Liang and Yuhua Chen

the geometric-arithmetic index, which is denoted as

$$
G A(G)=\sum_{u v \in E(G)} \frac{2 \sqrt{d(u) d(v)}}{d(u)+d(v)},
$$

where $d(u)$ denotes the degree of vertex $u \in V(G)$.

Yuan et al., [8] obtained the lower and upper bounds for geometric-arithmetic index of molecular graphs in terms of the numbers of vertices and edges. Furthermore, the $n$-vertex molecular trees with the minimum, the second and the third minimum, as well as the second and the third maximum geometric-arithmetic indices were determined. Das et al., [9] gave lower and upper bounds on geometric-arithmetic indices and characterized molecular graphs for which these bounds are best possible. Moreover, they discussed the effect on geometric-arithmetic index of inserting an edge into a molecular graph. Madanshekaf and Moradi [10] calculated the geometric-arithmetic index of two infinite classes of dendrimers.

Recently, Fath-Tabar et al., [11] developed a new version of the geometric -arithmetic index, i.e., the second geometric-arithmetic index:

$$
G A_{2}(G)=\sum_{u v E(G)} \frac{2 \sqrt{n(u) n(v)}}{n(u)+n(v)},
$$

where for each $u v \in E(G), n(u)$ is the number of vertices closer to vertex $u$ than vertex $v$ and $n(v)$ defines similarly. In Zhan and Qiao [12], the maximum and the minimum second geometric-arithmetic index of the star-like tree are learned in view of an increasing or decreasing transformation of the second geometric arithmetic index of trees, and the corresponding extremal trees are manifested.

Hasani et al., [13] introduced Co-PI index as

$$
C o-P I_{v}(G)=\sum_{u v \in E(G)}|n(u)-n(v)| .
$$

Su et al., [14] revealed that

$$
C o-P I_{v}(G)=\sum_{u v \in E(G)}|T(u)-T(v)|,
$$

where $T(u)=\sum_{v \in V(G)} d(u, v)$.

Although there have been several advances in geometric-arithmetic index and PI index of molecular graphs, the study of second geometric-arithmetic index and Co-PI index of special chemical structures is limited. In addition, as widespread and critical chemical structures, polyomino system, hexagonal system, V-phenylenic nanotubes and nanotori, and hexagonal triangle structure are widely used in medical science and pharmaceutical field. For example, as one of the basic chemical structures, polyomino chain exists widely in benzene and alkali molecular structures. Also, these indices are used for QSPR/QSAR study of organic compounds. For instance, QSAR study on acute toxicities of phenylsulfonyl carboxylate compounds, and QSPR/QSAR study on the water solubility and biological toxicity for fatty alcohol compounds. For these reasons, tremendous academic and industrial interests are attracted to research the second geometric-arithmetic index, Co-PI index and their extensional versions of special molecular structure from a mathematical point of view.

The contributions of our paper are five-fold. First, we give the second 
On Second Geometric-Arithmetic Index and Co-PI Index of Special Chemical Molecular Structures

geometric-arithmetic index and Co-PI index of unilateral polyomino chain. Second, the second geometric-arithmetic index and Co-PI index of unilateral hexagonal chain are calculated. Third, we derive these two indices of V-phenylenic nanotubes and nanotori. Fourth, two indices of hexagonal triangle are determined. At last, we extend these two indices into general version. The computational formulas of special chemical structure in general version are deduced.

\section{Indices of polyomino chain}

From the view in graph theory, polymino is a finite 2-connected planar graph and each interior face is surrounded by a square with length 4 . Polyomino chain is one class of polyomino such that the connection of centres for adjacent squares constitute a path $c_{1} c_{2} \cdots c_{n}$, where $c_{i}$ is the centre of $i$-th square. Polyomino chain $H_{n}^{4}$ is called a linear chain if the subgraph induced by all 3-degree vertices is a graph with $n-2$ squares. Furthermore, polyomino chain $H_{n}^{4}$ is called a Zig-zag chain if the subgraph induced by all vertices with degree $>2$ is path with $n-1$ edges. In what follows, we use $L_{n}^{4}$ and $Z_{n}^{4}$ to denote linear polyomino chain and Zig-zag polyomino chain, respectively. The structure of $L_{n}^{4}$ and $Z_{n}^{4}$ can refer to Figure 1. The purpose of this section is to obtain the second geometric-arithmetic index and Co-PI index of unilateral polyomino chain.

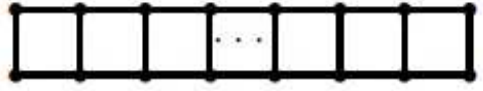

Linear polyomino chain

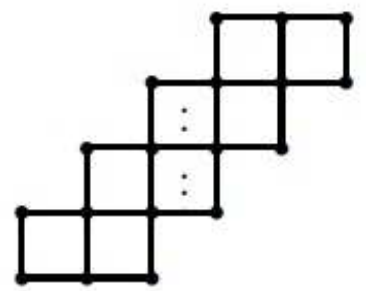

Zig-zag polyomino chain

Figure 1: The structure of $L_{n}^{4}$ and $Z_{n}^{4}$

The technologies we used here follow from Gao and Wang [5]. Choose an edge $e$ of the polyomino system and draw a straight line through the center of $e$, orthogonal on $e$ . This line will intersect the perimeter in two end points $P_{1}$ and $P_{2}$. The straight line segment $C$ whose end points are $P_{1}$ and $P_{2}$ is the elementary cut, intersecting the edge $e$. A fragment $S$ in polyomino chain is just maximal linear chain which includes the squares in start and end vertices. Let $l(S)$ be the length of fragment which denotes the number of squares it is contained. Let $H_{n}^{4}$ be a polyomino chain with $n$ squares and consist of fragment sequence $S_{1}, S_{2}, \cdots, S_{m}(m \geq 1)$. Denote $l\left(S_{i}\right)=l_{i}(i=1, \cdots, m)$. It is not difficult to verify that $l_{1}+l_{2}+\cdots+l_{m}=n+m-1$ and $\left|V\left(H_{n}^{4}\right)\right|=2 n+2$, $\left|E\left(H_{n}^{4}\right)\right|=3 n+1$. For the $k$-th fragment of polyomino chain, the cut of this fragment is 


\section{Wei Gao, Li Liang and Yuhua Chen}

the cut which intersects with $l_{k}+1$ parallel edges of squares in this fragment. A fragment called horizontal fragment if its cut parallels to the horizontal direction, and called vertical fragment if its cut parallels to the vertical direction. Unilateral polyomino chain is a special kind of polyomino chain such that for each vertical fragment, two horizontal fragments (if exists) adjacent it appear in the left and right sides, respectively.

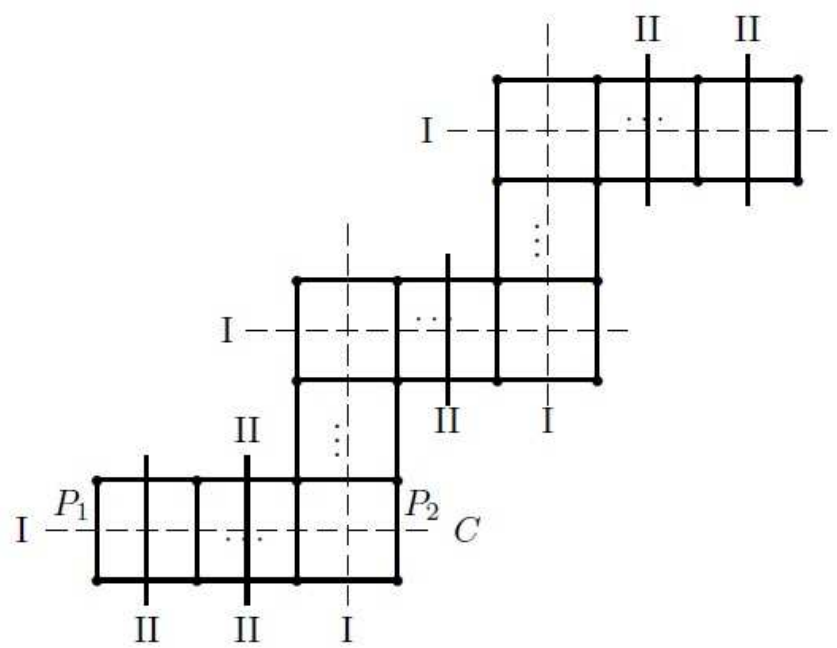

Figure 2: I-type cut and II-type cut of unilateral polyomino chain

The cuts in $H_{n}^{4}$ are divided into two types: I-type and II-type (see Figure 2). An edge is called I-type (or, II-type) if it intersects with I-type (or, II-type) cut. By virtue of intermediate results in Gao and Wang [5], we summarize the following facts.

- If edge $e=u v$ is I-type in $j$-th square of $k$-th fragment (i.e., $e$ is edge which is passed by dotted line in Figure 2), then

$$
n(u)= \begin{cases}l_{1}+1, & \text { if } k=1 \\ l_{m}+1, & \text { if } k=m \\ 2 \sum_{i=1}^{k-1} l_{i}-2 k+l_{k}+3, & \text { if } 2 \leq k \leq m-1,\end{cases}
$$

and

$$
n(v)= \begin{cases}2 \sum_{i=2}^{m} l_{i}-2 m+l_{1}+3, & \text { if } k=1 \\ 2 \sum_{i=1}^{m-1} l_{i}-2 m+l_{m}+3, & \text { if } k=m \\ 2 \sum_{i=k+1}^{m} l_{i}-2(m-k)+l_{k}+1, & \text { if } 2 \leq k \leq m-1 .\end{cases}
$$

- If edge $e$ is II-type in $j$-th square of $k$-th fragment (i.e., $e$ is edge which is passed by real line in Figure 2). Then 
On Second Geometric-Arithmetic Index and Co-PI Index of Special Chemical Molecular Structures

$$
n(u)= \begin{cases}2 j, & \text { if } k=1 \\ 2 l_{m}-2 j+2, & \text { if } k=m \\ 2 \sum_{i=1}^{k-1} l_{i}-2 k+2 j+2, & \text { if } 2 \leq k \leq m-1,\end{cases}
$$

and

$$
n(v)= \begin{cases}2 \sum_{i=2}^{m} l_{i}-2 m+2\left(l_{1}-j\right)+4, & \text { if } k=1 \\ 2 \sum_{i=1}^{m-1} l_{i}-2 m+2 j+2, & \text { if } k=m \\ 2 \sum_{i=k+1}^{m} l_{i}-2(m-k)+2\left(l_{k}-j\right)+2, & \text { if } 2 \leq k \leq m-1 .\end{cases}
$$

In view of above results and the definition of second geometric-arithmetic index and Co-PI index, our first result is stated as follows.

Theorem 1. Let $H_{n}^{4}$ be a unilateral polyomino chain consisted of $m$ fragment $S_{1}$, $S_{2}, \cdots, S_{m}(m \geq 1)$, and $l\left(S_{i}\right)=l_{i}(i=1, \cdots, m)$ be the length of each fragment. Then, we have

$$
\begin{aligned}
& G A_{2}\left(H_{n}^{4}\right)=2 \sum_{j=1}^{l_{1}-1} \frac{2 \sqrt{j\left(\sum_{i=1}^{m} l_{i}-m-j+2\right)}}{n+1}+2 \sum_{j=2}^{l_{m}} \frac{2 \sqrt{\left(\sum_{i=1}^{m-1} l_{i}-m+j+1\right)\left(l_{m}-j+1\right)}}{n+1} \\
& +2 \sum_{k=2}^{m-1} \sum_{j=2}^{l_{k}-1} \frac{2 \sqrt{\left(\sum_{i=1}^{k-1} l_{i}-k+j+1\right)\left(\sum_{i=k}^{m} l_{i}-(m-k)-j+1\right)}}{n+1} \\
& +\sum_{k=1}^{m}\left(l_{k}+1\right) \frac{\sqrt{\left(2 \sum_{i=1}^{k-1} l_{i}-2 k+l_{k}+3\right)\left(2 \sum_{i=k+1}^{m} l_{i}-2(m-k)+l_{k}+1\right)}}{n+1} \text {. } \\
& \operatorname{Co}-P I_{v}\left(H_{n}^{4}\right)=2 \sum_{j=1}^{l_{1}-1}\left|2 \sum_{i=1}^{m} l_{i}-2 m+4-4 j\right|+2 \sum_{j=2}^{l_{m}}\left|2 \sum_{i=1}^{m-1} l_{i}-2 m+4 j-2 l_{m}\right| \\
& +2 \sum_{k=2}^{m-1} \sum_{j=2}^{l_{k}-1}\left|2 \sum_{i=1}^{k-1} l_{i}-4 k+4 j-2 \sum_{i=k}^{m} l_{i}+2 m\right| \\
& +\sum_{k=1}^{m}\left(l_{k}+1\right)\left|2 \sum_{i=1}^{k-1} l_{i}-4 k+2-2 \sum_{i=k+1}^{m} l_{i}+2 m\right| \text {. }
\end{aligned}
$$


Wei Gao, Li Liang and Yuhua Chen

Corollary 2. Let $L_{n}^{4}$ be the linear chain with $n$ squares. Then, we have

$$
\begin{gathered}
G A_{2}\left(L_{n}^{4}\right)=2 \sum_{j=1}^{n-1} \frac{2 \sqrt{j(n+1-j)}}{n+1}+n+1, \\
C o-P I_{v}\left(L_{n}^{4}\right)=2 \sum_{j=1}^{n-1}|2 n+2-4 j| .
\end{gathered}
$$

Proof: By the definition of linear chain, we have $m=1, l_{1}=n, l_{2}=\cdots=l_{m}=0$. In terms of Theorem 1, we immediately get the result.

Corollary 3. Let $Z_{n}^{4}$ be the Zig-zag chain with $n$ squares. Then, we have

$$
\begin{gathered}
G A_{2}\left(Z_{n}^{4}\right)=\frac{4 \sqrt{m+1}}{m+2}+\frac{4 \sqrt{m+1}}{m+2}+\sum_{k=1}^{m} \frac{3 \sqrt{(2 k+1)(2 m-2 k+3)}}{m+2}, \\
C o-P I_{v}\left(Z_{n}^{4}\right)=8 m+\sum_{k=1}^{m} 3|4 k-2 m-2| .
\end{gathered}
$$

Proof: By virtue of the definition of Zig-zag chain, we have $m=n-1$, and $l_{1}=l_{2}=\cdots=l_{m}=2$. In view of Theorem 1 , the result is immediately obtained.

\section{Indices of hexagonal chain}

In this section, we report the second geometric-arithmetic index and Co-PI index of unilateral hexagonal chain.

Hexagonal chain is one class of hexagonal system which consisted by hexagonal. In hexagonal chain, each two hexagonals has one common edge or no common vertex. Two hexagonals are adjacented if they have common edge. No three or more hexagonals share one vertex. Each hexagonal has two adjacent hexagonals except hexagonals in terminus, and each hexagonal chain has two hexagonals in terminus. It is easy to verify that the hexagonal chain with $n$ hexagonals has $4 n+2$ vertices and $5 n+1$ edges. Let $L_{n}^{6}$ and $Z_{n}^{6}$ be the linear hexagonal chain and Zig-zag hexagonal chain, respectively. The chemical structure of $L_{n}^{6}$ and $Z_{n}^{6}$ can refer to Figure 3 for more detail.

The tricks we used in this section are presented in Gao and Wang [5]. Choose an edge $e$ of the hexagonal system and draw a straight line through the center of $e$, orthogonal on $e$. This line will intersect the perimeter in two end points $P_{1}$ and $P_{2}$. The straight line segment $C$ whose end points are $P_{1}$ and $P_{2}$ is the elementary cut, intersecting the edge $e$. A fragment $S$ in hexagonal chain is just maximal linear chain which include the hexagonals in start and end vertices. Let $l^{\prime}(S)$ be the length of fragment which denotes the number of hexagonals it is contained. Let $H_{n}^{6}$ be a hexagonal chain with $n$ hexagonals and consist of fragment sequence $S_{1}, S_{2}, \cdots, S_{m}(m \geq 1)$. Denote $l^{\prime}\left(S_{i}\right)=l_{i}^{\prime}(i=1, \cdots, m)$. Then, we verify that $l_{1}^{\prime}+l_{2}^{\prime}+\cdots+l_{m}^{\prime}=n+m-1$ since each two adjacent fragment have one common hexagonal. For the $k$-th fragment of 
On Second Geometric-Arithmetic Index and Co-PI Index of Special Chemical Molecular Structures

hexagonal chain, the cut of this fragment is the cut which intersects with $l_{k}^{\prime}+1$ parallel

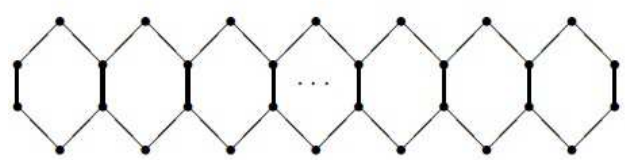

Linear hexagonal chain

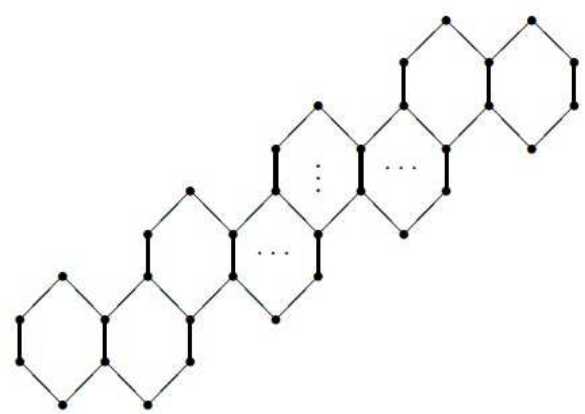

Zig-zag hexagonal chain

Figure 3: The structure of $L_{n}^{6}$ and $Z_{n}^{6}$

edges of hexagonals in this fragment. A fragment called horizontal fragment if its cut parallels to the horizontal direction, otherwise called inclined fragment. Unilateral hexagonal chain is a special class of hexagonal chain such that the cut for each inclined fragment at the same angle with a horizontal direction. As an example, Figure 4 shows a structure of unilateral hexagonal chain. Clearly, linear hexagonal chain $L_{n}^{6}$ is a unilateral hexagonal chain with one fragment, and Zig-zag is a unilateral hexagonal chain with $n-1$ fragments.

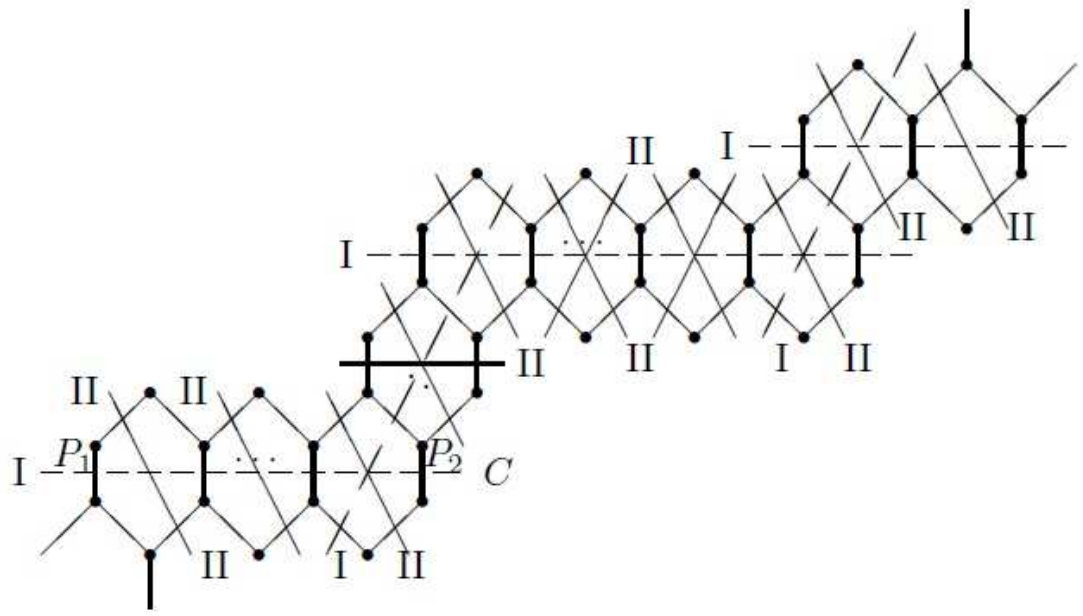

Figure 4. I-type cut and II-type cut of unilateral hexagonal chain

The cuts in $H_{n}^{6}$ are divided into two types: I-type and II-type (see Figure 4). A edge is called I-type if it intersects with I-type cut. Also, a edge is called II-type if it intersects with II-type cut. In terms of intermediate result of Gao and Wang [5], we sum up the following facts.

- If edge $e=u v$ is I-type in $j$-th square of $k$-th fragment (i.e., $e$ is edge which is 
Wei Gao, Li Liang and Yuhua Chen

passed by dotted line in Figure 4), then

$$
n(u)= \begin{cases}2 l_{1}^{\prime}+1, & \text { if } k=1 \\ 2 l_{m}^{\prime}+1, & \text { if } k=m \\ 4 \sum_{i=1}^{k-1}\left(l_{i}^{\prime}-k+1\right)+2 l_{k}^{\prime}+1, & \text { if } 2 \leq k \leq m-1,\end{cases}
$$

and

$$
n(v)= \begin{cases}4 \sum_{i=2}^{m}\left(l_{i}^{\prime}-m+1\right)+2 l_{1}^{\prime}+1, & \text { if } k=1 \\ 4 \sum_{i=1}^{m-1}\left(l_{i}^{\prime}-m+1\right)+2 l_{k}^{\prime}+1, & \text { if } k=m \\ 4 \sum_{i=k+1}^{m}\left(l_{i}^{\prime}-m+k\right)+2 l_{k}^{\prime}+1, & \text { if } 2 \leq k \leq m-1 .\end{cases}
$$

- If edge $e=u v$ is II-type in $j$-th square of $k$-th fragment (i.e., $e$ is edge which is passed by real line in Figure 4), then

$$
n(u)= \begin{cases}4 j-1, & \text { if } k=1 \\ 4 l_{m}^{\prime}-4 j+3, & \text { if } k=m \\ 4 \sum_{i=1}^{k-1}\left(l_{i}^{\prime}-k+1\right)+4 j-1, & \text { if } 2 \leq k \leq m-1,\end{cases}
$$

and

$$
n(v)= \begin{cases}4 \sum_{i=1}^{m} l_{i}^{\prime}-4 j+3, & \text { if } k=1 \\ 4 \sum_{i=1}^{m-1}\left(l_{i}^{\prime}-k+1\right)+4 j-1, & \text { if } k=m \\ 4 \sum_{i=k}^{m} l_{i}^{\prime}-4 j+3, & \text { if } 2 \leq k \leq m-1 .\end{cases}
$$

Using the definition of second geometric-arithmetic index and Co-PI index, we now show the computational formulas for unilateral hexagonal chain.

Theorem 4. Let $H_{n}^{6}$ be a unilateral hexagonal chain consisted of $m$ fragment $S_{1}, S_{2}$, $\cdots, S_{m}(m \geq 1)$, and $l^{\prime}\left(S_{i}\right)=l_{i}^{\prime}(i=1, \cdots, m)$ be the length of each fragment. Then, we have

$$
G A_{2}\left(H_{n}^{6}\right)=4 \sum_{j=1}^{i_{1}^{\prime}-1} \frac{\sqrt{(4 j-1)\left(4\left(\sum_{i=1}^{m} l_{i}^{\prime}-m+1\right)-4 j+3\right)}}{2 n+1}
$$


On Second Geometric-Arithmetic Index and Co-PI Index of Special Chemical Molecular Structures

$$
\begin{aligned}
& +2 \sum_{k=1}^{m-1} \frac{\sqrt{\left[4\left(\sum_{i=1}^{k} l_{i}^{\prime}-k+1\right)-1\right]\left[4\left(\sum_{i=k+1}^{m} l_{i}^{\prime}-m+k\right)+3\right]}}{2 n+1} \\
& +4 \sum_{k=2}^{m-1} \sum_{j=2}^{l_{k}^{-1}} \frac{\sqrt{\left[4\left(\sum_{i=1}^{k-1} l_{i}^{\prime}-k+1\right)+4 j-1\right]\left[4\left(\sum_{i=k}^{m} l_{i}^{\prime}-m+k\right)-4 j+3\right]}}{2 n+1} \\
& +4 \sum_{j=2}^{l_{m}^{\prime}} \frac{\sqrt{\left[4\left(\sum_{i=1}^{m-1} l_{i}^{\prime}-m+1\right)+4 j-1\right]\left(4 l_{m}^{\prime}-4 j+3\right)}}{2 n+1} \\
& +\sum_{k=2}^{m-1}\left(l_{k}^{\prime}+1\right) \frac{\sqrt{\left[4\left(\sum_{i=1}^{k-1} l_{i}^{\prime}-k+1\right)+2 l_{k}^{\prime}+1\right]\left[4\left(\sum_{i=k+1}^{m} l_{i}^{\prime}-m+k\right)+2 l_{k}^{\prime}+1\right]}}{2 n+1} \\
& C o-P I_{v}\left(H_{n}^{6}\right)=4 \sum_{j=1}^{i_{1}^{\prime}-1}\left|4 \sum_{i=1}^{m} l_{i}^{\prime}-4 m-8 j+8\right|+2 \sum_{k=1}^{m-1}\left|4 \sum_{i=1}^{k} l_{i}^{\prime}-8 k-4 \sum_{i=k+1}^{m} l_{i}^{\prime}+4 m\right| \\
& +4 \sum_{k=2}^{m-1} \sum_{j=2}^{l_{k}-1}\left|4 \sum_{i=1}^{k-1} l_{i}^{\prime}-8 k+8 j-4 \sum_{i=k}^{m} l_{i}^{\prime}+4 m\right|+4 \sum_{j=2}^{i_{m}^{\prime}}\left|4 \sum_{i=1}^{m-1} l_{i}^{\prime}-4 m+8 j-4 l_{m}^{\prime}\right| \\
& +\sum_{k=2}^{m-1}\left(l_{k}^{\prime}+1\right)\left|4 \sum_{i=1}^{k-1} l_{i}^{\prime}-8 k-4 \sum_{i=k+1}^{m} l_{i}^{\prime}+4 m+4\right| .
\end{aligned}
$$

Corollary 5. Let $L_{n}^{6}$ be the linear chain with $n$ hexagonals. Then, we have

$$
\begin{gathered}
G A_{2}\left(L_{n}^{6}\right)=4 \sum_{j=1}^{n-1} \frac{\sqrt{(4 j-1)(4 n-4 j+3)}}{2 n+1}+4 \sum_{j=2}^{n} \frac{\sqrt{(4 j-1)(4 n-4 j+3)}}{2 n+1}, \\
C o-P I_{v}\left(L_{n}^{6}\right)=4 \sum_{j=1}^{n-1}|4 n-8 j+4|+4 \sum_{j=2}^{n}|4 n-8 j+4| .
\end{gathered}
$$

Proof: By the definition of linear chain, we check that $m=1, l_{1}^{\prime}=n, l_{2}^{\prime}=\cdots=l_{m}^{\prime}=0$. In terms of Theorem 4, we get the result soon.

Corollary 6. Let $Z_{n}^{6}$ be the Zig-zag chain with $n$ hexagonals. Then, we have

$$
\begin{gathered}
G A_{2}\left(Z_{n}^{6}\right)=8 \frac{\sqrt{3(4 m+3)}}{2 m+3}+2 \sum_{k=1}^{m-1} \frac{\sqrt{(4 k+3)(4 m-4 k+3)}}{2 m+3}+\sum_{k=2}^{m-1} 3 \frac{\sqrt{(4 k+1)(4 m-4 k+5)}}{2 m+3}, \\
C o-P I_{v}\left(Z_{n}^{6}\right)=32 m+2 \sum_{k=1}^{m-1}|8 k-4 m|+\sum_{k=2}^{m-1} 3|8 k-4 m-4| .
\end{gathered}
$$


Wei Gao, Li Liang and Yuhua Chen

Proof: Using the definition of Zig-zag chain, we verify that $m=n-1$ and $l_{1}^{\prime}=l_{2}^{\prime}=\cdots=l_{m}^{\prime}=2$. In view of Theorem 4 , the corollary is immediately yielded.

\section{Indices of V-Phenylenic nanotubes and nanotori}

The notations in this section follow from Diudea [15]. The molecular structures V-Phenylenic nanotube and V-Phenylenic nanotorus are denoted by $\operatorname{VPHX}[m, n]$ and $V P H Y[m, n]$, respectively. The structures of $V P H X[m, n]$ and $V P H Y[m, n]$ are described in Figure 5 an Figure 6, respectively.

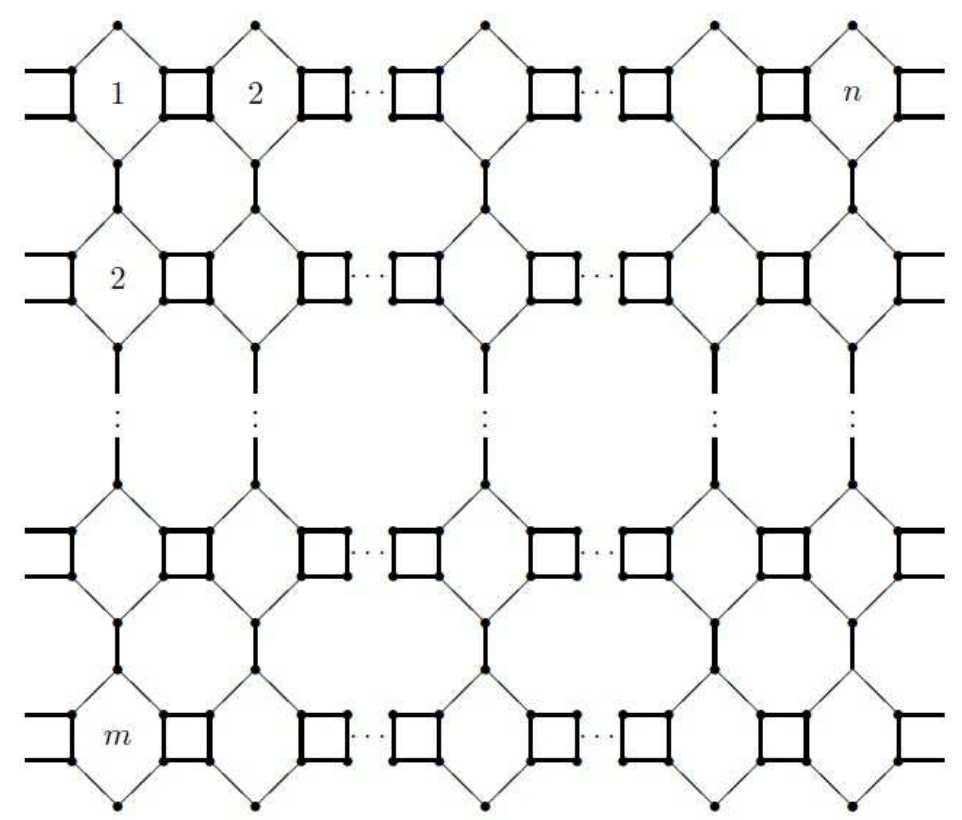

Figure 5: The structure of V-phenylenic nanotube

Let $\beta=\min \{n, m\}$ and $S_{i}=3+9+15+\cdots+(6 i-3)$. Based on the tricks described in Gao and Wang [5], we show the the representations of $G A_{2}(V P H X[m, n])$ and $C o-P I_{v}(V P H X[m, n])$ in Theorem 7, and the expressions of $G A_{2}(V P H Y[m, n])$ and $C o-P I_{v}(V P H Y[m, n])$ are determined in Theorem 8.

Theorem 7.

$$
\begin{gathered}
G A_{2}(V P H X[m, n])= \begin{cases}\alpha_{1}, & \text { if } m \neq n \\
\alpha_{2}, & \text { if } m=n .\end{cases} \\
\text { Co-PI }(V P H X[m, n])= \begin{cases}\alpha_{3}, & \text { if } m \neq n \\
\alpha_{4}, & \text { if } m=n .\end{cases}
\end{gathered}
$$

Here, $\alpha_{i}(1 \leq i \leq 4)$ are given as follows: 
On Second Geometric-Arithmetic Index and Co-PI Index of Special Chemical Molecular Structures

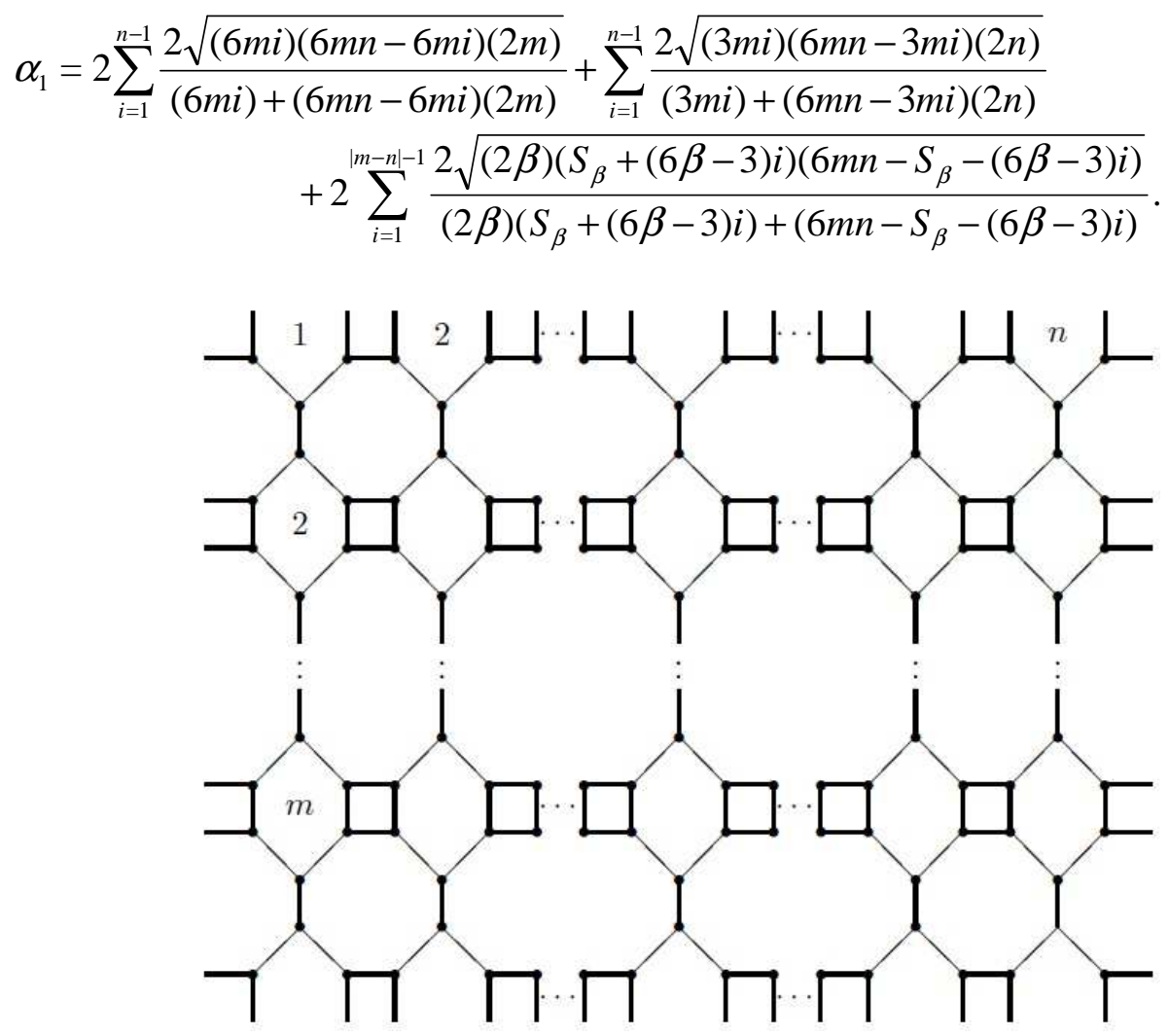

Figure 6: The structure of V-phenylenic nanotorus

$$
\begin{aligned}
& \alpha_{2}=2 \sum_{i=1}^{n-1} \frac{2 \sqrt{(6 m i)(6 m n-6 m i)(2 m)}}{(6 m i)+(6 m n-6 m i)(2 m)}+\sum_{i=1}^{n-1} \frac{2 \sqrt{(3 m i)(6 m n-3 m i)(2 n)}}{(3 m i)+(6 m n-3 m i)(2 n)} \\
& +\frac{2 \sqrt{4 n\left(S_{n}+6 n-3\right)\left(6 m n-S_{n}-(6 n-3)\right)}}{4 n\left(S_{n}+6 n-3\right)+\left(6 m n-S_{n}-(6 n-3)\right)} \text {. } \\
& \alpha_{3}=2 \sum_{i=1}^{n-1}|(6 m i)-(6 m n-6 m i)(2 m)|+\sum_{i=1}^{n-1}|(3 m i)-(6 m n-3 m i)(2 n)| \\
& +2 \sum_{i=1}^{|m-n|-1}\left|(2 \beta)\left(S_{\beta}+(6 \beta-3) i\right)-\left(6 m n-S_{\beta}-(6 \beta-3) i\right)\right| \text {. } \\
& \alpha_{4}=2 \sum_{i=1}^{n-1}|(6 m i)-(6 m n-6 m i)(2 m)|+\sum_{i=1}^{n-1}|(3 m i)-(6 m n-3 m i)(2 n)| \\
& +\left|4 n\left(S_{n}+6 n-3\right)-\left(6 m n-S_{n}-(6 n-3)\right)\right| \text {. }
\end{aligned}
$$


Wei Gao, Li Liang and Yuhua Chen

Theorem 8.

$$
\begin{gathered}
G A_{2}(V P H Y[m, n])= \begin{cases}\alpha_{5}, & \text { if } m \neq n \\
\alpha_{6}, & \text { if } m=n .\end{cases} \\
\text { Co-PI }(V P H Y[m, n])= \begin{cases}\alpha_{7}, & \text { if } m \neq n \\
\alpha_{8}, & \text { if } m=n .\end{cases}
\end{gathered}
$$

Here, $\alpha_{i}(5 \leq i \leq 8)$ are described as follows:

$$
\begin{aligned}
& \alpha_{5}=2 \sum_{i=1}^{n-1} \frac{2 \sqrt{(6 m i)(6 m n-6 m i)(2 m)}}{(6 m i)+(6 m n-6 m i)(2 m)}+2 \sum_{i=1}^{n-1} \frac{2 \sqrt{(3 m i)(6 m n-3 m i)(2 n)}}{(3 m i)+(6 m n-3 m i)(2 n)} \\
& +4 \sum_{i=1}^{|m-n|-1} \frac{2 \sqrt{(2 \beta)\left(S_{\beta}+(6 \beta-3) i\right)\left(6 m n-S_{\beta}-(6 \beta-3) i\right)}}{(2 \beta)\left(S_{\beta}+(6 \beta-3) i\right)+\left(6 m n-S_{\beta}-(6 \beta-3) i\right)} . \\
& \alpha_{6}=2 \sum_{i=1}^{n-1} \frac{2 \sqrt{(6 m i)(6 m n-6 m i)(2 m)}}{(6 m i)+(6 m n-6 m i)(2 m)}+2 \sum_{i=1}^{n-1} \frac{2 \sqrt{(3 m i)(6 m n-3 m i)(2 n)}}{(3 m i)+(6 m n-3 m i)(2 n)} \\
& +\frac{2 \sqrt{4 n\left(S_{n}+6 n-3\right)\left(6 m n-S_{n}-(6 n-3)\right)}}{4 n\left(S_{n}+6 n-3\right)+\left(6 m n-S_{n}-(6 n-3)\right)} \text {. } \\
& \alpha_{7}=2 \sum_{i=1}^{n-1}|(6 m i)-(6 m n-6 m i)(2 m)|+2 \sum_{i=1}^{n-1}|(3 m i)-(6 m n-3 m i)(2 n)| \\
& +4 \sum_{i=1}^{|m-n|-1}\left|(2 \beta)\left(S_{\beta}+(6 \beta-3) i\right)-\left(6 m n-S_{\beta}-(6 \beta-3) i\right)\right| \text {. } \\
& \alpha_{8}=2 \sum_{i=1}^{n-1}|(6 m i)-(6 m n-6 m i)(2 m)|+2 \sum_{i=1}^{n-1}|(3 m i)-(6 m n-3 m i)(2 n)| \\
& +\left|4 n\left(S_{n}+6 n-3\right)-\left(6 m n-S_{n}-(6 n-3)\right)\right| \text {. }
\end{aligned}
$$

\section{Indices of Hexagonal triangle graph}

In this section, we determine the second geometric-arithmetic index and Co-PI index of hexagonal triangle graph $T(n)$. This molecular graph is a class of nanotube, and the structure of $T(n)$ can refer to Fig 7 which is related to the atomic structure of bipod shaped nanocrystals.

Theorem 9. Let $T(n)$ be hexagonal triangle graph with $n$ rows. Then, we yield

$$
\begin{gathered}
G A_{2}(T(n))=3 \sum_{1 \leq i \leq n}(i+1) \frac{2 \sqrt{\left(i^{2}+2 i\right)\left(n^{2}+4 n+1-i^{2}-2 i\right)}}{n^{2}+4 n+1} \\
C o-P I_{v}(T(n))=3 \sum_{1 \leq i \leq n}(i+1)\left|n^{2}+4 n+1-2 i^{2}-4 i\right|
\end{gathered}
$$


On Second Geometric-Arithmetic Index and Co-PI Index of Special Chemical Molecular Structures

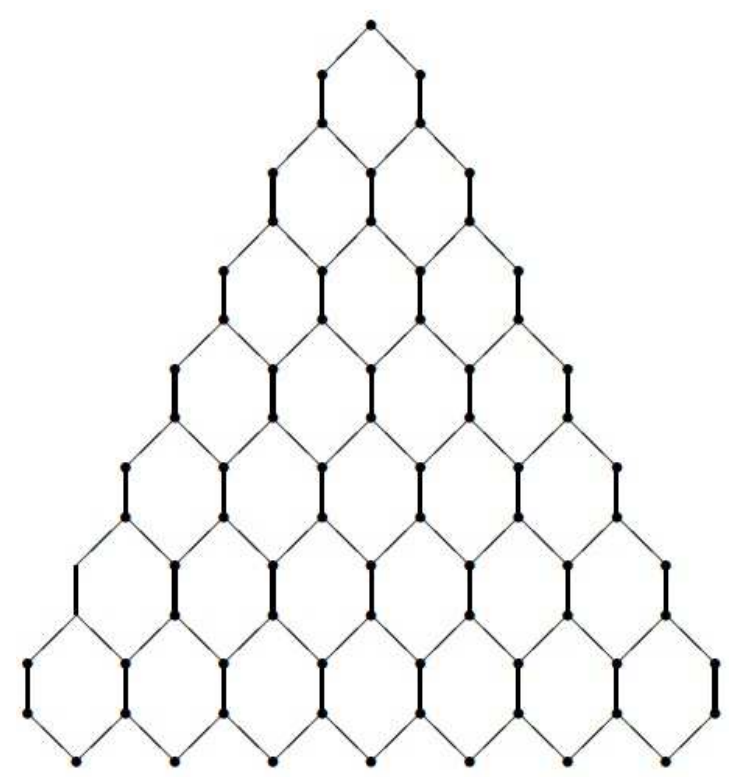

Figure 7: The structure of hexagonal triangle graph.

Proof: By observing the structure of hexagonal triangle graph, we find that there are $i$ hexagons in the $i$-th row, where $1 \leq i \leq n$. Since $T(n)$ has an equilateral figure, we get $|E(T(n))|=\frac{3}{2}\left(n^{2}+3 n\right)$ and $|V(T(n))|=n^{2}+4 n+1$. Furthermore, $i$-th row has exactly $i+1$ vertical edges of hexagonal triangle graph. Let $e=u v$ be an edge in $i$-th row, we deduce

$$
n(u)=i^{2}+2 i
$$

and

$$
n(v)=n^{2}+4 n+1-i^{2}-2 i
$$

Thus, by virtue of the definition, we get

$$
\begin{aligned}
& G A_{2}(T(n))=3 \sum_{1 \leq i \leq n}(i+1) \frac{2 \sqrt{n(u) n(v)}}{n(u)+n(v)} \\
& =3 \sum_{1 \leq i \leq n}(i+1) \frac{2 \sqrt{\left(i^{2}+2 i\right)\left(n^{2}+4 n+1-i^{2}-2 i\right)}}{n^{2}+4 n+1}, \\
& \text { and } \\
& C o-P I_{v}(T(n))=3 \sum_{1 \leq i \leq n}(i+1)|n(u)-n(v)|=3 \sum_{1 \leq i \leq n}(i+1)\left|n^{2}+4 n+1-2 i^{2}-4 i\right| .
\end{aligned}
$$

Thus, the desired results are obtained.

\section{General Second geometric-arithmetic index and general Co-PI index}

For the requirement of chemical applications, the indices should be expended into more 
Wei Gao, Li Liang and Yuhua Chen

generalized forms. For example, Li and Gutman [16] extended the ordinary Randic connectivity index to general Randic connectivity index, and Zhou and Trinajstic [17] raised general sum-connectivity index which extended the sum-connectivity index.

In this section, we expend the definition of second geometric-arithmetic index and Co-PI index. The general second geometric-arithmetic index is defined as:

$$
G A_{2}^{\gamma}(G)=\sum_{u v \in(G)}\left(\frac{2 \sqrt{n(u) n(v)}}{n(u)+n(v)}\right)^{\gamma},
$$

where $\gamma$ is a real number. Also, we introduce general Co-PI index as

$$
C o-P I_{v}^{\gamma}(G)=\sum_{u v \in E(G)}|n(u)-n(v)|^{\gamma},
$$

where $\gamma$ is a real number. By the results in Su et al., [14], we infer

$$
C o-P I_{v}^{\gamma}(G)=\sum_{u v \in E(G)}|T(u)-T(v)|^{\gamma} .
$$

Evidently, $G A_{2}^{1}$ and $C o-P I_{v}^{1}$ are the second geometric-arithmetic index and Co-PI index, respectively.

By virtue of the results determined in former sections, we get the following extended conclusions.

Theorem 10. Let $H_{n}^{4}$ be a unilateral polyomino chain consisted of $m$ fragment $S_{1}, S_{2}$ , $\cdots, S_{m}(m \geq 1)$, and $l\left(S_{i}\right)=l_{i}(i=1, \cdots, m)$ be the length of each fragment. Then, for any real number $\gamma$, we yield

$$
\begin{gathered}
G A_{2}^{\gamma}\left(H_{n}^{4}\right)=2 \sum_{j=1}^{l_{1}-1}\left(\frac{2 \sqrt{j\left(\sum_{i=1}^{m} l_{i}-m-j+2\right)}}{n+1}\right)^{\gamma} \\
+2 \sum_{j=2}^{l_{m}}\left(\frac{2 \sqrt{\left(\sum_{i=1}^{m-1} l_{i}-m+j+1\right)\left(l_{m}-j+1\right)}}{n+1}\right)^{\gamma} \\
+2 \sum_{k=2}^{m-1} \sum_{j=2}^{l_{k}-1}\left(\frac{2 \sqrt{\left(\sum_{i=1}^{k-1} l_{i}-k+j+1\right)\left(\sum_{i=k}^{m} l_{i}-(m-k)-j+1\right)}}{n+1}\right)^{\gamma} \\
+\sum_{k=1}^{m}\left(l_{k}+1\right)\left(\frac{\sqrt{\left(2 \sum_{i=1}^{k-1} l_{i}-2 k+l_{k}+3\right)\left(2 \sum_{i=k+1}^{m} l_{i}-2(m-k)+l_{k}+1\right)}}{n+1}\right)^{\gamma} . \\
\text { Co-PI } I_{v}^{\gamma}\left(H_{n}^{4}\right)=2 \sum_{j=1}^{l_{1}-1}\left|2 \sum_{i=1}^{m} l_{i}-2 m+4-4 j\right|^{\gamma}
\end{gathered}
$$


On Second Geometric-Arithmetic Index and Co-PI Index of Special Chemical Molecular Structures

$$
\begin{aligned}
& +2 \sum_{j=2}^{l_{m}}\left|2 \sum_{i=1}^{m-1} l_{i}-2 m+4 j-2 l_{m}\right|^{\gamma} \\
& +2 \sum_{k=2}^{m-1} \sum_{j=2}^{l_{k}-1}\left|2 \sum_{i=1}^{k-1} l_{i}-4 k+4 j-2 \sum_{i=k}^{m} l_{i}+2 m\right|^{\gamma} \\
& +\sum_{k=1}^{m}\left(l_{k}+1\right)\left|2 \sum_{i=1}^{k-1} l_{i}-4 k+2-2 \sum_{i=k+1}^{m} l_{i}+2 m\right|^{\gamma} .
\end{aligned}
$$

Corollary 11. Let $L_{n}^{4}$ be the linear chain with $n$ squares. Then, for any real number $\gamma$, we deduce

$$
\begin{gathered}
G A_{2}^{\gamma}\left(L_{n}^{4}\right)=2 \sum_{j=1}^{n-1}\left(\frac{2 \sqrt{j(n+1-j)}}{n+1}\right)^{\gamma}+n+1, \\
C o-P I_{v}^{\gamma}\left(L_{n}^{4}\right)=2 \sum_{j=1}^{n-1}|2 n+2-4 j|^{\gamma} .
\end{gathered}
$$

Corollary 12. Let $Z_{n}^{4}$ be the Zig-zag chain with $n$ squares. Then, for any real number $\gamma$, we infer

$$
\begin{gathered}
G A_{2}^{\gamma}\left(Z_{n}^{4}\right)=2\left(\frac{2 \sqrt{m+1}}{m+2}\right)^{\gamma}+2\left(\frac{2 \sqrt{m+1}}{m+2}\right)^{\gamma}+\sum_{k=1}^{m} 3\left(\frac{\sqrt{(2 k+1)(2 m-2 k+3)}}{m+2}\right)^{\gamma} \\
C o-P I_{v}^{\gamma}\left(Z_{n}^{4}\right)=4(2 m)^{\gamma}+\sum_{k=1}^{m} 3|4 k-2 m-2|^{\gamma} .
\end{gathered}
$$

Theorem 13. Let $H_{n}^{6}$ be a unilateral hexagonal chain consisted of $m$ fragment $S_{1}, S_{2}$ , $\cdots, S_{m}(m \geq 1)$, and $l^{\prime}\left(S_{i}\right)=l_{i}^{\prime}(i=1, \cdots, m)$ be the length of each fragment. Then, for any real number $\gamma$, we obtain

$$
\begin{aligned}
& G A_{2}^{\gamma}\left(H_{n}^{6}\right)=4 \sum_{j=1}^{i_{1}^{\prime}-1}\left(\frac{\sqrt{(4 j-1)\left[4\left(\sum_{i=1}^{m} l_{i}^{\prime}-m+1\right)-4 j+3\right]}}{2 n+1}\right)^{\gamma} \\
& +2 \sum_{k=1}^{m-1}\left(\frac{\sqrt{\left[4\left(\sum_{i=1}^{k} l_{i}^{\prime}-k+1\right)-1\right]\left[4\left(\sum_{i=k+1}^{m} l_{i}^{\prime}-m+k\right)+3\right]}}{2 n+1}\right)^{\gamma} \\
& +4 \sum_{k=2}^{m-1}\left(\sum_{j=2}^{i_{k}^{\prime}-1} \frac{\sqrt{\left[4\left(\sum_{i=1}^{k-1} l_{i}^{\prime}-k+1\right)+4 j-1\right]\left[4\left(\sum_{i=k}^{m} l_{i}^{\prime}-m+k\right)-4 j+3\right]}}{2 n+1}\right)^{\gamma}
\end{aligned}
$$


Wei Gao, Li Liang and Yuhua Chen

$$
\begin{aligned}
& +4 \sum_{j=2}^{i_{m}^{\prime}}\left(\frac{\sqrt{\left[4\left(\sum_{i=1}^{m-1} l_{i}^{\prime}-m+1\right)+4 j-1\right]\left(4 l_{m}^{\prime}-4 j+3\right)}}{2 n+1}\right)^{\gamma} \\
& +\sum_{k=2}^{m-1}\left(l_{k}^{\prime}+1\right)\left(\frac{\sqrt{\left[4\left(\sum_{i=1}^{k-1} l_{i}^{\prime}-k+1\right)+2 l_{k}^{\prime}+1\right]\left[4\left(\sum_{i=k+1}^{m} l_{i}^{\prime}-m+k\right)+2 l_{k}^{\prime}+1\right]}}{2 n+1}\right)^{\gamma} . \\
& \operatorname{Co}-P I_{v}^{\gamma}\left(H_{n}^{6}\right)=4 \sum_{j=1}^{i_{1}^{\prime}-1}\left|4 \sum_{i=1}^{m} l_{i}^{\prime}-4 m-8 j+8\right|^{\gamma} \quad+2 \sum_{k=1}^{m-1}\left|4 \sum_{i=1}^{k} l_{i}^{\prime}-8 k-4 \sum_{i=k+1}^{m} l_{i}^{\prime}+4 m\right|^{\gamma} \\
& +4 \sum_{k=2}^{m-1} \sum_{j=2}^{i_{k}^{\prime}-1}\left|4 \sum_{i=1}^{k-1} l_{i}^{\prime}-8 k+8 j-4 \sum_{i=k}^{m} l_{i}^{\prime}+4 m\right|^{\gamma} \quad+4 \sum_{j=2}^{i_{m}^{\prime}}\left|4 \sum_{i=1}^{m-1} l_{i}^{\prime}-4 m+8 j-4 l_{m}^{\prime}\right|^{\gamma} \\
& +\sum_{k=2}^{m-1}\left(l_{k}^{\prime}+1\right)\left|4 \sum_{i=1}^{k-1} l_{i}^{\prime}-8 k-4 \sum_{i=k+1}^{m} l_{i}^{\prime}+4 m+4\right|^{\gamma} .
\end{aligned}
$$

Corollary 14. Let $L_{n}^{6}$ be the linear chain with $n$ hexagonals. Then, for any real number $\gamma$, we have

$$
\begin{gathered}
G A_{2}^{\gamma}\left(L_{n}^{6}\right)=4 \sum_{j=1}^{n-1}\left(\frac{\sqrt{(4 j-1)(4 n-4 j+3)}}{2 n+1}\right)^{\gamma}+4 \sum_{j=2}^{n}\left(\frac{\sqrt{(4 j-1)(4 n-4 j+3)}}{2 n+1}\right)^{\gamma}, \\
C o-P I_{v}^{\gamma}\left(L_{n}^{6}\right)=4 \sum_{j=1}^{n-1}|4 n-8 j+4|^{\gamma}+4 \sum_{j=2}^{n}|4 n-8 j+4|^{\gamma} .
\end{gathered}
$$

Corollary 15. Let $Z_{n}^{6}$ be the Zig-zag chain with $n$ hexagonals. Then, for any real number $\gamma$, we get $G A_{2}^{\gamma}\left(Z_{n}^{6}\right)=8\left(\frac{\sqrt{3(4 m+3)}}{2 m+3}\right)^{\gamma}+2 \sum_{k=1}^{m-1}\left(\frac{\sqrt{(4 k+3)(4 m-4 k+3)}}{2 m+3}\right)^{\gamma}$,

$$
\begin{gathered}
+\sum_{k=2}^{m-1} 3\left(\frac{\sqrt{(4 k+1)(4 m-4 k+5)}}{2 m+3}\right)^{\gamma} \\
C o-P I_{v}^{\gamma}\left(Z_{n}^{6}\right)=8|4 m|^{\gamma}+2 \sum_{k=1}^{m-1}|8 k-4 m|^{\gamma}+\sum_{k=2}^{m-1} 3|8 k-4 m-4|^{\gamma} .
\end{gathered}
$$

Theorem 16. For any real number $\gamma$, we infer

$$
G A_{2}^{\gamma}(V P H X[m, n])= \begin{cases}\alpha_{9}, & \text { if } m \neq n \\ \alpha_{10}, & \text { if } m=n .\end{cases}
$$


On Second Geometric-Arithmetic Index and Co-PI Index of Special Chemical Molecular Structures

$$
C o-P I_{v}^{\gamma}(V P H X[m, n])= \begin{cases}\alpha_{11}, & \text { if } m \neq n \\ \alpha_{12}, & \text { if } m=n .\end{cases}
$$

Here, $\alpha_{i}(9 \leq i \leq 12)$ is given as follows:

$$
\begin{aligned}
& \alpha_{9}=2 \sum_{i=1}^{n-1}\left(\frac{2 \sqrt{(6 m i)(6 m n-6 m i)(2 m)}}{(6 m i)+(6 m n-6 m i)(2 m)}\right)^{\gamma}+\sum_{i=1}^{n-1}\left(\frac{2 \sqrt{(3 m i)(6 m n-3 m i)(2 n)}}{(3 m i)+(6 m n-3 m i)(2 n)}\right)^{\gamma} \\
& +2 \sum_{i=1}^{|m-n|-1}\left(\frac{2 \sqrt{(2 \beta)\left(S_{\beta}+(6 \beta-3) i\right)\left(6 m n-S_{\beta}-(6 \beta-3) i\right)}}{(2 \beta)\left(S_{\beta}+(6 \beta-3) i\right)+\left(6 m n-S_{\beta}-(6 \beta-3) i\right)}\right)^{\gamma} . \\
& \alpha_{10}=2 \sum_{i=1}^{n-1}\left(\frac{2 \sqrt{(6 m i)(6 m n-6 m i)(2 m)}}{(6 m i)+(6 m n-6 m i)(2 m)}\right)^{\gamma}+\sum_{i=1}^{n-1}\left(\frac{2 \sqrt{(3 m i)(6 m n-3 m i)(2 n)}}{(3 m i)+(6 m n-3 m i)(2 n)}\right)^{\gamma} \\
& +\left(\frac{2 \sqrt{4 n\left(S_{n}+6 n-3\right)\left(6 m n-S_{n}-(6 n-3)\right)}}{4 n\left(S_{n}+6 n-3\right)+\left(6 m n-S_{n}-(6 n-3)\right)}\right)^{\gamma} \text {. } \\
& \alpha_{11}=2 \sum_{i=1}^{n-1}|(6 m i)-(6 m n-6 m i)(2 m)|^{\gamma}+\sum_{i=1}^{n-1}|(3 m i)-(6 m n-3 m i)(2 n)|^{\gamma} \\
& +2 \sum_{i=1}^{|m-n|-1}\left|(2 \beta)\left(S_{\beta}+(6 \beta-3) i\right)-\left(6 m n-S_{\beta}-(6 \beta-3) i\right)\right|^{\gamma} \text {. } \\
& \alpha_{12}=2 \sum_{i=1}^{n-1}|(6 m i)-(6 m n-6 m i)(2 m)|^{\gamma}+\sum_{i=1}^{n-1}|(3 m i)-(6 m n-3 m i)(2 n)|^{\gamma} \\
& +\left|4 n\left(S_{n}+6 n-3\right)-\left(6 m n-S_{n}-(6 n-3)\right)\right|^{\gamma} \text {. }
\end{aligned}
$$

Theorem 17. For any real number $\gamma$, we deduce

$$
\begin{gathered}
G A_{2}^{\gamma}(V P H Y[m, n])= \begin{cases}\alpha_{13}, & \text { if } m \neq n \\
\alpha_{14}, & \text { if } m=n .\end{cases} \\
C o-P I_{v}^{\gamma}(V P H Y[m, n])= \begin{cases}\alpha_{15}, & \text { if } m \neq n \\
\alpha_{16}, & \text { if } m=n .\end{cases}
\end{gathered}
$$

Here, $\alpha_{i}(13 \leq i \leq 16)$ is given as follows:

$$
\begin{aligned}
& \alpha_{13}=2 \sum_{i=1}^{n-1}\left(\frac{2 \sqrt{(6 m i)(6 m n-6 m i)(2 m)}}{(6 m i)+(6 m n-6 m i)(2 m)}\right)^{\gamma}+2 \sum_{i=1}^{n-1}\left(\frac{2 \sqrt{(3 m i)(6 m n-3 m i)(2 n)}}{(3 m i)+(6 m n-3 m i)(2 n)}\right)^{\gamma} \\
& +4 \sum_{i=1}^{|m-n|-1}\left(\frac{2 \sqrt{(2 \beta)\left(S_{\beta}+(6 \beta-3) i\right)\left(6 m n-S_{\beta}-(6 \beta-3) i\right)}}{(2 \beta)\left(S_{\beta}+(6 \beta-3) i\right)+\left(6 m n-S_{\beta}-(6 \beta-3) i\right)}\right)^{\gamma} . \\
& \alpha_{14}=2 \sum_{i=1}^{n-1}\left(\frac{2 \sqrt{(6 m i)(6 m n-6 m i)(2 m)}}{(6 m i)+(6 m n-6 m i)(2 m)}\right)^{\gamma}+2 \sum_{i=1}^{n-1}\left(\frac{2 \sqrt{(3 m i)(6 m n-3 m i)(2 n)}}{(3 m i)+(6 m n-3 m i)(2 n)}\right)^{\gamma}
\end{aligned}
$$


Wei Gao, Li Liang and Yuhua Chen

$$
\begin{gathered}
+\left(\frac{2 \sqrt{4 n\left(S_{n}+6 n-3\right)\left(6 m n-S_{n}-(6 n-3)\right)}}{4 n\left(S_{n}+6 n-3\right)+\left(6 m n-S_{n}-(6 n-3)\right)}\right)^{\gamma} . \\
\left.\alpha_{15=2 \sum_{i=1}^{n-1} \mid(6 m i)-}(6 m n-6 m i)(2 m)\right|^{\gamma}+2 \sum_{i=1}^{n-1}|(3 m i)-(6 m n-3 m i)(2 n)|^{\gamma} \\
+4 \sum_{i=1}^{|m-n|-1}\left|(2 \beta)\left(S_{\beta}+(6 \beta-3) i\right)-\left(6 m n-S_{\beta}-(6 \beta-3) i\right)\right|^{\gamma} . \\
\alpha_{16}=2 \sum_{i=1}^{n-1} \mid(6 m i)- \\
-\left.(6 m n-6 m i)(2 m)\right|^{\gamma}+2 \sum_{i=1}^{n-1}|(3 m i)-(6 m n-3 m i)(2 n)|^{\gamma} \\
+\left|4 n\left(S_{n}+6 n-3\right)-\left(6 m n-S_{n}-(6 n-3)\right)\right|^{\gamma} .
\end{gathered}
$$

Theorem 18. Let $T(n)$ be hexagonal triangle graph with $n$ rows. Then, for any real number $\gamma$, we derive

\section{Conclusion}

$$
\begin{gathered}
G A_{2}^{\gamma}(T(n))=3 \sum_{1 \leq i \leq n}(i+1)\left(\frac{2 \sqrt{\left(i^{2}+2 i\right)\left(n^{2}+4 n+1-i^{2}-2 i\right)}}{n^{2}+4 n+1}\right)^{\gamma} \\
C o-P I_{v}^{\gamma}(T(n))=3 \sum_{1 \leq i \leq n}(i+1)\left|n^{2}+4 n+1-2 i^{2}-4 i\right|^{\gamma}
\end{gathered}
$$

In this paper, we report the second geometric-arithmetic index and Co-PI index of several special chemical molecular structures, including unilateral polyomino chain, unilateral hexagonal chain, V-Phenylenic nanotube, V-Phenylenic nanotorus and hexagonal triangle graph. Furthermore, we introduce the general version of second geometric-arithmetic index and Co-PI index, and obtain corresponding results for these special chemical molecular structures. The results achieved in our article illustrates the promising application prospects in biology, pharmacy and chemical engineering.

Acknowledgments. We thank the reviewers for their constructive comments in improving the quality of this paper. We also would like to thank the anonymous referees for providing us with constructive comments and suggestions. The research is partially supported by NSFC (nos. 11401519, 11371328, and 11471293).

\section{REFERENCES}

1. L.Yan, Y.Li, W.Gao and J.Li, On the extremal hyper-wiener index of graphs, Journal of Chemical and Pharmaceutical Research, 6(3) (2014) 477-481.

2. W.Gao and L.Shi, Wiener index of gear fan graph and gear wheel graph, Asian Journal of Chemistry, 26(11) (2014) 3397-3400.

3. W.F.Xi and W.Gao, Geometric-arithmetic index and Zagreb indices of certain special molecular graphs, Journal of Advances in Chemistry, 10(2) (2014) 2254-2261.

4. J.Y.Dou, Y.Y.Wang and W.Gao, Some characteristics on hyper-wiener index of graphs, Journal of Chemical and Pharmaceutical Research, 6(5) (2014) 1659-1663.

5. W.Gao and W.F.Wang, Second atom-bond connectivity index of special chemical molecular structures, Journal of Chemistry, volume 2014, Article ID 906254, 8 pages, 
On Second Geometric-Arithmetic Index and Co-PI Index of Special Chemical Molecular Structures

http://dx.doi.org/10.1155/2014/906254.

6. J.A.Bondy, U.S.R.Mutry, Graph Theory, Spring, Berlin, 2008.

7. D.Vukicevic and B.Furtula, Topological index based on the ratios of geometrical and arithmetical means of end-vertex degrees of edges, Journal of Mathematical Chemistry, 4 (2009) 1369-1376.

8. Y.Yuan, B.Zhou and N.Trinajstic, On geometric-arithmetic index, Journal of Mathematical Chemistry, 47 (2010) 833-841.

9. K.C.Das, I.Gutman and B.Furtula, On the first geometric-arithmetic index of graphs, Discrete Applied Mathematics, 159 (2011) 2030-2037.

10. A.Madanshekaf and M.Moradi, The first geometric-arithmetic index of some nanostar dendrimers, Iranian Journal of Mathematical Chemistry, 5 (2014) (S1) 1-6.

11. G.F.Tabar, B.Purtula and I.Gutman, A new geometric-arithmetic index, Journal of Mathematical Chemistry, 47 (2010) 477-486.

12. F.Q.Zhan and Y.F.Qiao, The second geometric-arithmetic index of the starlike tree with $k$-component, Mathematics in Practice and Theory, 44(7) (2014) 226-229.

13. F.Hasani, O.Khormali and A.Iranmanesh, Computation of the first vertex of Co-PI index of $\mathrm{TUC} 4 \mathrm{C}(\mathrm{S})$ nanotubes, Optoelectronics and Advanced Materials-Rapid Communications, 4(4) (2010) 544-547.

14. G.Su, X.Xiong and X.Xu, On the Co-PI and laplacian Co-PI eigenvalues of graph, Discrete Applied Mathematics, 161 (2013) 277-293.

15. M.V.Diudea, Fullerenes, phenylenic and naphthylenic tori, Nanotubes and Carbon Nanostructures, 10(4) (2002) 273-292.

16. X.Li and I.Gutman, Mathematical Aspects of Randic-Type Molecular Structure Descriptors, University of Kragujevac, Kragujevac, 2006.

17. B.Zhou and N.Trinajstic, On general sum-connectivity index, Journal of Mathematical Chemistry, 47 (2010) 210-218. 